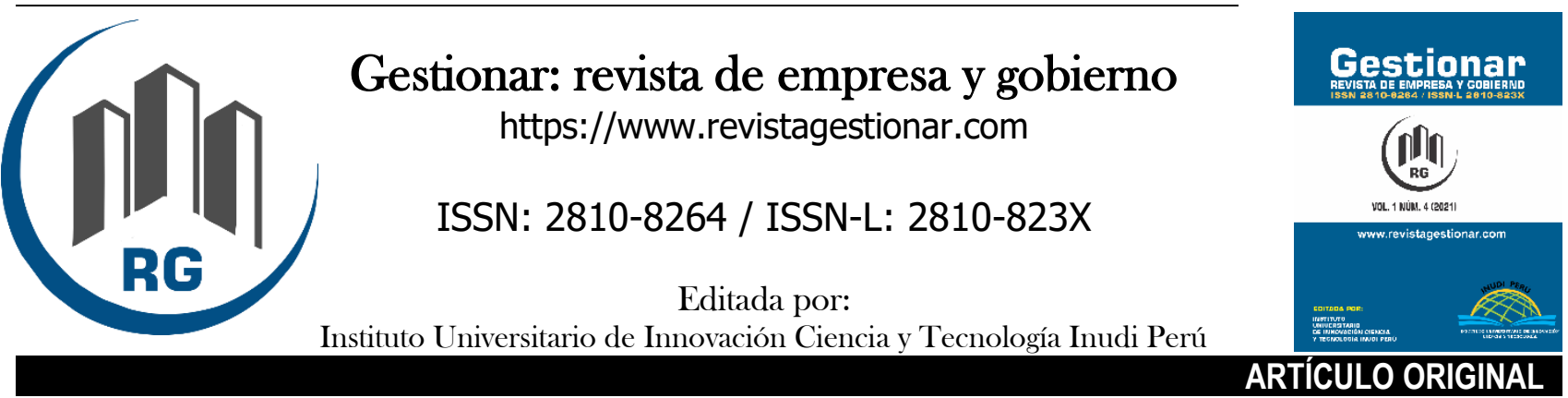

\title{
Formación docente, investigación e innovación en educación superior: caso de una universidad colombiana
}

\section{Teacher training, research and innovation in higher education: case of a Colombian university}

Formação docente, pesquisa e inovação no ensino superior: caso de uma universidade colombiana

\author{
Francisco Blanchar ${ }^{1}$ \\ Universidad de La Guajira, Riohacha - La Guajira, Colombia \\ (iD https://orcid.org/0000-0002-0176-8551 \\ franciscoblanchar@hotmail.com \\ Yadely Martínez \\ Universidad de La Guajira, Riohacha - La Guajira, Colombia \\ (iD https://orcid.org/0000-0003-1756-6492 \\ yademary@hotmail.com \\ DOl: https://doi.org/10.35622/j.rg.2022.01.001
}

Recibido: 24/10/2021 Aceptado: 28/01/2022 Publicado: 13/01/2022

PALABRAS CLAVE

alta calidad, educación superior, ingeniería industrial, innovación, investigación.

\section{KEYWORDS \\ high quality, higher education, industrial}

\begin{abstract}
RESUMEN. El objetivo del estudio fue determinar el nivel de efectividad del programa de Ingeniería Industrial de la Universidad de La Guajira, Colombia en materia de procesos de formación para la investigación, el espíritu crítico, creación. Así como por sus aportes al conocimiento científico, innovación, desarrollo cultural de acuerdo a lo dispuesto en el factor investigación, innovación, creación artística y cultural, en materia de acreditación en alta calidad. El diseño de la investigación fue de naturaleza cualitativa, bajo un diseño de estudio de caso, específicamente relacionado con los procesos, las interrelaciones y dinámicas del programa de Ingeniería Industrial en función de la labor docente y de los estudiantes para el fomento de la investigación, a fin de comprender los avances, persistencias y oportunidades de mejora. Se utilizaron como instrumentos, una entrevista semiestructurada, una ficha de registro y análisis documental. Los resultados permitieron establecer el cumplimiento en alto grado, por parte del programa, de los aspectos relacionados con las actividades en materia de la formación para la investigación, la innovación, la creación artística y cultural.
\end{abstract}

ABSTRACT. The study's objective was to determine the effectiveness of the Industrial Engineering program of the University of La Guajira, Colombia, in terms of training processes for research, critical spirit, creation. As well as for their contributions to scientific knowledge, innovation, cultural development following the research, innovation, artistic and cultural creation factor in terms of highquality accreditation. The research design was qualitative in nature, under a case study design,

\footnotetext{
${ }^{1}$ Doctorando en Ciencias de la Educación y Magister en educación de la Universidad Tecnológica de Pereira. Correspondencia: franciscoblanchar@hotmail.com
} 
engineering, innovation, research.

\section{PALAVRAS-CHAVE}

alta qualidade, ensino superior, engenharia industrial, inovação, pesquisa. specifically related to the processes, interrelationships, and dynamics of the Industrial Engineering program based on the teaching and student work to promote research, to understand the progress, persistence, and opportunities for improvement. A semi-structured interview, a registration form, and a documentary analysis were used as instruments. The results made it possible to establish a high degree of compliance, by the program, with aspects related to training for research, innovation, artistic and cultural creation.

RESUM0. 0 objetivo do estudo foi determinar o nível de eficácia do programa de Engenharia Industrial da Universidade de La Guajira, Colômbia em termos de processos de formação para pesquisa, espírito crítico, criação. Bem como pelos seus contributos para o conhecimento científico, inovação, desenvolvimento cultural de acordo com as disposições do fator de investigação, inovação, criação artística e cultural, em termos de acreditação de alta qualidade. 0 desenho da pesquisa foi de natureza qualitativa, sob um desenho de estudo de caso, especificamente relacionado aos processos, inter-relações e dinâmicas do curso de Engenharia Industrial com base no ensino e no trabalho dos alunos para a promoção da pesquisa, a fim de compreender o progresso, persistência e oportunidades de melhoria. Como instrumentos foram utilizados a entrevista semiestruturada, a ficha de registro e a análise documental. Os resultados permitiram estabelecer um alto grau de cumprimento, por parte do programa, dos aspectos relacionados às atividades relacionadas à formação para pesquisa, inovação, criação artística e cultural.

\section{INTRODUCCIÓN}

Uno de los pilares fundamentales para el desarrollo y progreso de las sociedades lo constituye el papel de sus ciudadanos, y la importancia del rol constructivo, propositivo, de resolución de problemas locales, regionales y mundiales derivados de sus capacidades como individuos, así como entes constituyentes de grupos sociales/colectivos. Ante este panorama la educación cumple un rol trascendental en la formación, desarrollo, consolidación de habilidades, competencias, aprendizajes y capacidades para la participación ciudadana en todas las esferas y dimensiones. Las instituciones educativas de carácter formal, informal y para el desarrollo humano deben desarrollar gestiones pertinentes para participar en el logro de estos objetivos. Principalmente el papel de las universidades, como rectoras de la educación superior y desarrollo de los sujetos para su participación en el mercado laboral, pilar de las economías, sistemas ambientales, sociales y financieros de las naciones.

Al respecto, la Organización de las Naciones Unidas para la Educación, la Ciencia y la Cultura UNESCO (2009) en eventos como la conferencia mundial sobre la educación superior manifiesta que, como en ningún momento de la historia, la educación superior representa un eslabón fundamental para el desarrollo y construcción de la sociedad. Para el logro de estos propósitos debe enmarcar sus aspectos misionales en actividades de investigación, enseñanza y aprendizajes pertinentes a las comunidades. Desde aristas adecuadas a la autonomía institucional de los centros formativos que denoten calidad de las acciones educativas, pertinencia al contexto, condiciones de su área de influencia, sujetos involucrados, transparencia en el actuar, gestión, administración de recursos de diversa índole, y sobre todo responsabilidad social con los grupos sociales atendidos y sus comunidades.

Ante esta dinámica mundial de la educación como proceso transformador y motor del desarrollo social, Colombia alineó política educativa, en materia de educación superior, con las tendencias formativas a nivel internacional, estableciendo rutas de gestión a través de sus diversas instituciones educativas. Principalmente desde el ente rector, Ministerio de Educación Nacional MEN en materia de estrategias para que los entes 
universitarios aporten a través de sus Proyectos Educativos Institucionales PEI en la disposición misional y los objetivos de una educación de calidad para la sociedad actual y del futuro.

La universidad de la Guajira, ente universitario insignia del departamento que lleva el mismo nombre, ha dispuesto toda su capacidad institucional para dar cuenta de las proyecciones mundiales, nacionales y regionales en materia de acreditación institucional. Ofrecer a la comunidad Guajira una educación pertinente, de alta calidad, acorde con las necesidades y derechos formativos de los usuarios del sistema de educación superior y la sociedad (Consejo Nacional de Acreditación [CNA], 2015).

Es así como el programa de Ingeniería Industrial de esta universidad, ha establecido la necesidad de aunar todos los recursos disponibles para materializar la gestión institucional y las directrices universitarias en un programa de pregrado alta calidad, el cual debe reconocerse. Como lo plantea el CNA (2013) en materia de sus procesos de investigación, innovación, creación artística y cultural "la efectividad en sus procesos de formación para la investigación, el espíritu crítico, la creación, sus aportes al conocimiento científico, a la innovación y al desarrollo cultural" (p. 47).

Con el fin de lograr el aporte referenciado, el presente trabajo de investigación plantea desarrollar un estudio analítico de la gestión del programa de Ingeniería Industrial de la Universidad de La Guajira en materia de los factores y características definidas por el modelo del Consejo Nacional de Acreditación, relacionados con el factor No. 6 "Investigación y creación artística y cultural". Destaca la necesidad de lograr la efectividad de los procesos de formación para la investigación, el espíritu crítico, la creación, los aportes al conocimiento científico, a la innovación y al desarrollo cultural. Este trabajo se basa en el análisis de la característica No. 29 de este factor.

Esta característica indaga por la capacidad del programa para promover la capacidad de indagación y búsqueda, y la formación de un espíritu investigativo, creativo e innovador en aras de contribuir al favorecimiento de los estudiantes y lograr una aproximación crítica y permanente de estos al estado del arte en el área de conocimiento del programa.

El siguiente interrogante central de la investigación ¿Cuál es el nivel de efectividad del programa de Ingeniería Industrial de la Universidad de la Guajira en materia de los procesos de formación para la investigación, el espíritu crítico, la creación, sus aportes al conocimiento científico, a la innovación y al desarrollo cultural?

El objetivo general fue determinar el nivel de efectividad del programa de Ingeniería Industrial de la Universidad de la Guajira en materia de los procesos de formación para la investigación, el espíritu crítico, la creación, sus aportes al conocimiento científico, la innovación y al desarrollo cultural de acuerdo a lo dispuesto en el factor investigación, innovación, creación artística y cultural establecido por el CNA en materia del proceso de autoevaluación institucional. Los objetivos específicos: a) Describir la naturaleza de los procesos de formación para la investigación, el espíritu crítico y la creación, y por sus aportes al conocimiento científico, a la innovación y al desarrollo cultural por parte del programa de Ingeniería Industrial de la Universidad de La Guajira; b) Determinar la capacidad de indagación y búsqueda, y la formación de un espíritu investigativo, creativo e innovador del programa de Ingeniería Industrial para favorecer una aproximación crítica y permanente de los 
estudiantes y c) Analizar la organización organizativa del programa en materia del núcleo de profesores adscritos y su relación con el tiempo significativo dedicado a la investigación, a la innovación y a la creación artística y cultural.

\section{MÉTODO Y MATERIALES}

\section{Tipo de investigación}

La investigación fue de naturaleza cualitativa, entendida como un paradigma de estudio sistemático de la realidad, que busca su comprensión e interpretación profunda, en ámbitos diversos como la educación y los contextos sociales, escenarios socioeducativos y demás entornos relevantes para el ser humano. Esto permite tomar de decisiones en función del descubrimiento y desarrollo de un cuerpo organizado de conocimiento (Sandín, 2003). En este caso se trata de comprender y determinar el nivel de efectividad del programa de Ingeniería Industrial de la Universidad de la Guajira en materia de los procesos de formación para la investigación y la innovación.

\section{Técnica de investigación}

La investigación se circunscribe a partir de un Estudio de Caso. El cual es definido por Stake (1999) como un proceso de los aspectos particulares y complejos de un caso singular, con el objetivo de comprender su actividad en circunstancias importantes. El caso aborda los procesos, las interrelaciones y dinámicas del programa de Ingeniería Industrial a fin de comprender sus avances, persistencias y oportunidades de mejora.

\section{Técnicas e instrumentos de recolección de información}

Preliminarmente se diseñó e implementó un consentimiento informado (ver anexo 1). Donde se informa a los actores participantes (director del programa, docentes y estudiantes) de los objetivos, propósitos de la investigación, confidencialidad y uso exclusivamente académico de los datos e información recolectada a partir de los instrumentos diseñados.

Posteriormente al consentimiento, se procedió a implementar los instrumentos respectivos, validados por el juicio de expertos de los jurados de la investigación.

Técnica de la Entrevista. Instrumento Guía de Entrevista Semiestructurada. Considerada como una técnica de gran utilidad en la investigación cualitativa, dada su utilidad para recabar datos. Se entiende como una conversación entre el investigador y el sujeto de estudio, a fin de obtener respuestas verbales a las interrogantes planteadas sobre el problema propuesto (Díaz et al., 2013). Implementada para obtener la perspectiva de tres actores del programa de Ingeniería Industrial de Uniguajira acerca del proceso de autoevaluación y acreditación del programa: director del programa, docentes (2) y estudiantes (2).

El guion de entrevista consta de 19 interrogantes orientadores diseñadas en torno a las categorías y subcategorías de análisis (ver anexo 2).

Técnica de Análisis documental. Instrumento Ficha de registro y análisis documental. Se trata de una técnica de recuperación de información que responde a las necesidades informativas de los usuarios. A partir de la cual se pretende conocer segmentos específicos de información de algún documento en particular y en la medida 
de las posibilidades, conocer la totalidad de información relevante que exista sobre un tema específico (Vickery, 1970).

Ficha de registro y análisis documental de fuentes primarias y secundarias, internas y externas, relacionadas con los procesos académicos del programa de Ingeniería Industrial (ver anexo 3).

\section{Unidad de análisis}

La investigación establece como unidad de análisis, la efectividad del programa de Ingeniería Industrial de Uniguiajira en materia de los procesos de formación para la investigación, el espíritu crítico y la creación (CNA, 2013).

En la tabla 1 se define la categoría y las subcategorías de análisis implicadas en el estudio.

Tabla 1.

Categoría de análisis

\begin{tabular}{ll}
\hline Categorías & Subcategorías \\
\hline 1.1. Capacidad de promoción de la indagación, creatividad e innovación. \\
Entendida como los criterios, estrategias y actividades del programa, \\
orientados a promover la capacidad de indagación y búsqueda, y la \\
formación de un espíritu investigativo, creativo e innovador en los \\
estudiantes.
\end{tabular}

1.2. Existencia y uso de mecanismos de fomento de ideas de investigación por parte de profesores. Da cuenta de la existencia y utilización de mecanismos por parte de los profesores adscritos al programa, para incentivar en los estudiantes la generación de ideas y problemas de investigación.

Formación para la investigación, la innovación y la creación artística y cultural

1.3. Actividad estudiantil en monitorias e investigación. Estudiantes que están vinculados como monitores, auxiliares de investigación e integrantes de semilleros y/o grupos de investigación.

1.4. Grupos y semilleros de investigación. Nivel de participación estudiantil en grupos y semilleros de investigación del programa, de acuerdo con su tipo y modalidad.

1.5. Actividades académicas investigativas. Tipo de cursos electivos, seminarios, pasantías, eventos, derivados de líneas de investigación durante los últimos cinco años.

1.6. Actividades académicas empresariales. Tipo de pasantías, talleres, actividades conjuntas- relacionadas con la realidad empresarial.

1.7. Vinculación Plan de estudios-Sector productivo. Existencia dentro del plan de estudios de espacios académicos y de vinculación con el sector 
productivo. Se analiza la naturaleza de la investigación científica, técnica y tecnológica, la innovación.

1.8. Participación estudiantil en investigación. Involucramiento de los estudiantes en los programas institucionales de jóvenes investigadores.

1.9. Participación estudiantil en prácticas empresariales. Intervención de los estudiantes en prácticas empresariales en temas de investigación y desarrollo, ingeniería y experimentación en Colombia y en el exterior.

1.10. Participación estudiantil en proyectos universidad-empresa-Estado. Participación de los estudiantes en proyectos Universidad-Empresa-Estado que adelante la Institución.

1.11. Participación estudiantil en programas de innovación. Participación de los estudiantes en programas de innovación tales como transferencia de conocimiento, emprendimiento y creatividad.

\section{Unidad de trabajo}

La unidad de trabajo se compone de dos tipos de elementos: a) actores académicos (el director de programa, dos docentes y dos estudiantes) del programa de Ingeniería Industrial de la Universidad de La Guajira y b) Fuentes documentales que soportan las evidencias de los procesos académicos asociados con el factor 6 del modelo de acreditación de programas de pregrado (CNA, 2013).

\section{RESULTADOS}

El factor investigación, innovación y creación artística y cultural da cuenta de las características de un programa de pregrado de alta calidad orientado hacia la efectividad en sus procesos de formación para la investigación, el espíritu crítico, la creación, y por sus aportes al conocimiento científico, a la innovación y al desarrollo cultural. A partir de dos características centrales, alusivas a su cuerpo docente y el componente estudiantil: a) la contribución en materia de formación para la investigación, la innovación, la creación artística y cultural.

En la tabla 3 se presentan los resultados generales derivados del proceso de calificación del factor 6 , en materia del análisis y estudio del desempeño del programa de ingeniería industrial de Uniguajira, en los dos periodos recientes (2016 y 2021).

Tabla 3.

Calificación y grado de cumplimiento del factor 6 durante los procesos de autoevaluación 2016 y 2021.

\begin{tabular}{lllllll}
\hline \multirow{2}{*}{ Factor } & \multicolumn{2}{l}{ Ponderación } & \multicolumn{3}{l}{$\begin{array}{l}\text { Calificación } \\
\text { (sobre 100 puntos) }\end{array}$} & \multicolumn{2}{l}{ Grado de cumplimiento } \\
\cline { 2 - 7 } & 2016 & 2021 & 2016 & 2021 & 2016 & 2021 \\
\hline $\begin{array}{l}\text { Factor 6. } \\
\text { Investigación, }\end{array}$ & 10,20 & 10,20 & 84,66 & 88,20 & $\begin{array}{l}\text { Se cumple en } \\
\text { alto grado }\end{array}$ & $\begin{array}{l}\text { Se cumple en } \\
\text { alto grado }\end{array}$ \\
\hline
\end{tabular}


Innovación, Creación

Artística y Cultural

Fuente: Adaptación del informe de resultados de la autoevaluación del programa de Ingeniería Industrial con fines de Acreditación (Uniguajira, 2018).

De manera complementaria a los resultados generales, en la tabla 4 se presenta la ponderación de la característica relacionada durante los periodos de análisis.

Tabla 4.

Calificación y grado de cumplimiento de las características del factor 6 durante los procesos de autoevaluación 2016 y 2021.

\begin{tabular}{|c|c|c|c|c|c|c|}
\hline \multirow[t]{2}{*}{ Característica } & \multicolumn{2}{|c|}{ Ponderación } & \multicolumn{2}{|c|}{$\begin{array}{l}\text { Calificación } \\
\text { (sobre } 100 \text { puntos) }\end{array}$} & \multicolumn{2}{|c|}{ Grado de cumplimiento } \\
\hline & 2016 & 2021 & 2016 & 2021 & 2016 & 2021 \\
\hline Característica $\quad$ No. 29. & & & & & & \\
\hline $\begin{array}{l}\text { Formación para la } \\
\text { investigación, la } \\
\text { innovación, la creación } \\
\text { artística y cultural. }\end{array}$ & 57,40 & 57,40 & 86,97 & 87,28 & $\begin{array}{lr}\text { Se } & \text { cumple } \\
\text { en } & \text { alto } \\
\text { grado } & \end{array}$ & $\begin{array}{l}\text { Se cumple en } \\
\text { alto grado }\end{array}$ \\
\hline
\end{tabular}

Fuente: Adaptación del informe de resultados de la autoevaluación del programa de Ingeniería Industrial con fines de Acreditación (Uniguajira, 2018) y elaboración propia.

Los resultados establecidos a partir del análisis de las categorías relacionadas indican la evolución y desarrollo positivos del compromiso institucional, el esfuerzo a nivel de la facultad de ingenierías. La disposición y cumplimiento direccionado del programa de ingeniería industrial de la Universidad de La Guajira en materia de procesos de formación para la investigación, el espíritu crítico y la creación, y por sus aportes al conocimiento científico, a la innovación y al desarrollo cultural.

\section{Formación para la investigación, la innovación y la creación artística y cultural (característica 29). Subcategoría Capacidad de promoción de la indagación, creatividad e innovación.}

En primera instancia, a nivel de las políticas, programas, proyectos y estrategias a nivel institucional, se destaca el papel del programa por participar en los procesos de en materia de acreditación, autoevaluación y autorregulación institucional. En este marco, la dinámica se ha orientado en incorporar las directrices del sistema de investigación institucional, particularmente mediante el comité de investigación y extensión de la facultad.

Sumado a la articulación programa-facultad-sistema de investigación, se destaca el trabajo programático del plan de estudios del programa, su revisión, valoración, análisis de la pertinencia y disposición curricular en materia de promoción de la investigación y la innovación. Abarca la definición y establecimiento de asignaturas clave en función de las dinámicas internacionales, nacionales e institucionales que propendan por el desarrollo investigativo y la consolidación de competencias científicas en los estudiantes. Además de la definición, actualización periódica de las líneas de investigación institucionales dada la realidad, el contexto del área de influencia atendida por el ente universitario y el panorama de la sociedad frente al programa. Finalmente, la 
configuración de propuestas, proyectos, estudios contemporáneos y pertinentes acordes a las líneas investigativas vigentes.

\section{Subcategoría existencia y uso de mecanismos de fomento de ideas de investigación por parte de profesores.}

El trabajo en función del trabajo docente a partir de rutas metodológicas accesibles para el fomento de la investigación, principalmente vinculada con el sector empresarial del contexto próximo a los estudiantes y los grupos sociales de los cuales hacen parte. A manera de garantizar desde el programa la planificación, desarrollo, evaluación de prácticas de clases, proyectos de investigación, extensión en cada asignatura vinculada, su proyección hacia las realidades, requerimientos de las comunidades y entornos industriales de referencia.

Dentro de las estrategias contempladas por la facultad y el programa para garantizar dinámicas exitosas en materia de la promoción de la investigación desde el trabajo docente, se encuentra el sistema de evaluación electrónica del desempeño docente. Esta consiste en una iniciativa de la Facultad de Ingeniería de la Universidad de La Guajira para registrar, sistematizar y analizar las evidencias de la evaluación del desempeño docente. El proceso contempla los procesos de autoevaluación y heteroevaluación en línea, bajo la participación de los docentes y estudiantes, referidos a los objetivos institucionales y del programa específico, entre los cuales se encuentra los procesos de investigación e innovación.

\section{Subcategoría Actividad estudiantil en monitorias e investigación}

Al respecto, el programa da cuenta de las Políticas y estrategias sobre estímulos académicos para los estudiantes de Uniguajira, que en su artículo 14 establece el otorgamiento de estímulos académicos, entre otros aspectos, la mención meritoria o laureada de trabajos de grado y de monitorias. Adicionalmente se contempla el desarrollo de vinculación estudiantil como tutores académicos, auxiliares de investigación y pasantes en prácticas académicas a nivel nacional e internacional, como intercambios y prácticas.

Este tipo de actividades, dinamizadas desde el programa, tiene como objetivo la ampliación, complementariedad, profundización y enriquecimiento de los conocimientos teóricos, prácticos y metodológicos derivados de la formación profesional, entendidos bajo la modalidad denominada "Movilidad estudiantil", establecida mediante el Acuerdo 006 de 2016 (Uniguajira, 2016). La cual enmarca una serie de programas de fomento de la participación de los estudiantes en intercambios, pasantías o prácticas por periodos hasta de un año, en Colombia 0 en el exterior. En contraprestación, la universidad permite la participación de estudiantes provenientes de otros entes universitarios.

\section{Subcategoría Grupos y semilleros de investigación}

El programa ha establecido la iniciativa de promover la participación en grupos y semilleros de investigación, en tanto diversos esfuerzos:

A nivel institucional: Se destaca la expedición del Estatuto de Investigación (Universidad de La Guajira, 2021b) como directriz general, que determina, entre otros aspectos, la importancia de los grupos, semilleros de investigación, como entidades colectivas estructurales y funcionales del denominado Sistema de Investigación de la Universidad. 
En materia de los grupos de investigación, estos son definidos como uno de los organismos directivos del Comité Central de Investigación y Extensión de la Universidad (Capítulo V, artículo 11). Además, se establece que cinco representantes de estos grupos, institucionalmente constituidos, hacen parte de los Comités de investigación y extensión de las facultades (Capítulo V, artículo 16). Puntualmente del artículo 22 al artículo 25 se determina la definición y el mecanismo de creación de los grupos y semilleros de investigación en la universidad de La Guajira, respectivamente.

El estatuto abarca las directrices del sistema de investigación de la universidad en materia de los principios, las políticas, objetivos y estrategias de la investigación. Considera también las políticas, objetivos, estrategias y liderazgo de la Vicerrectoría de investigación y extensión (objetivo, misión y visión), del Sistema de Investigación, sus organismos directivos, de la línea de investigación, los proyectos, normas para tramitación de informes y financiación, la evaluación de proyectos, presentación de informes parciales, investigadores, estímulos, obligaciones y convocatorias de docentes investigadores y el Fondo especial de investigaciones de las convocatorias internas del centro de documentación y publicaciones.

A nivel del programa Ingeniería Industrial se ha logrado dinamizar acciones de consolidación de los grupos de investigación, bajo la participación de estudiantes y docentes a nivel interno y en los procesos de categorización. A nivel del programa, se destaca la participación estudiantil en los 20 grupos de investigación adscritos a la Facultad de Ingenierías hasta el año 2020 (Ministerio de Ciencias, 2018).

\section{Subcategoría Actividades académicas empresariales}

Se destaca el trabajo de la Facultad de Ingenierías, y específicamente del programa mediante el establecimiento de convenios a mediano y largo plazo con las principales asociaciones empresariales del departamento de La Guajira, entidades de carácter nacional (181 convenios) e internacional (43 convenios).

\section{Subcategoría Vinculación Plan de estudios-Sector productivo}

El plan de estudios vigente del programa de Ingeniería Industrial contempla 7 asignaturas específicamente relacionadas con el fortalecimiento y consolidación de una cultura de la investigación. En el sentido de tener entre sus propósitos principales la formación de profesionales competitivos e innovadores, que participen desde las primeras fases y periodos de formación académica en grupos de investigación, semilleros, investigaciones y procesos de indagación científica. Además de proveer las condiciones pertinentes para su crecimiento personal, profesional y laboral mediante actividades prácticas en el sector productivo relacionado con sus intereses. Las asignaturas relacionadas con las siguientes:

Gestión Tecnológica e Innovación y Electiva V, dos asignaturas vinculadas con los procesos asociados a la tecnología, sus usos, importancia e incidencia en las dinámicas académicas y empresariales del mundo contemporáneo, la necesidad de conocimientos de la innovación y la evolución de este tipo de recursos.

Proyecto de Investigación I, II y III, asignaturas enmarcadas en la consolidación progresiva de habilidades y competencias científicas en los estudiantes, en función del proceso dinámico de formulación y socialización de proyectos de investigación, originados en las propuestas, materializados en los anteproyectos y consolidados en los informes finales de investigación. Este proceso se sustenta en las actividades de los grupos de 
investigación institucionalizados desde la facultad y el programa, y se basa en el trabajo colectivo entre el estudiante, el docente director, compañeros pares académicos y expertos evaluadores.

Emprendimiento empresarial y formulación y evaluación de proyectos, asignaturas orientadoras para la formación investigativa empresarial y el emprendimiento, en función del análisis y formulación de planes de negocios pertinentes, viables y materializables, acorde a los intereses de los estudiantes, las necesidades de la realidad social, económica y ambiental de la región y el país, y el entorno empresarial contemporáneo.

\section{Subcategoría Participación estudiantil en prácticas empresariales}

Con el fin de consolidar el trabajo institucional en el marco del fomento de la participación de los estudiantes en prácticas empresariales en temas de investigación y desarrollo, ingeniería y experimentación en Colombia y en el exterior, Uniguajira ha establecido, mantenido y desarrollado un sinnúmero de convenios para prácticas en ámbitos empresariales a nivel nacional e internacional.

Tabla 5. Entidades de convenios para prácticas empresariales

\begin{tabular}{cl}
\hline Item & Empresa \\
\hline 1 & Carbones del Cerrejón Limited \\
3 & Alcaldía del Municipio de Villanueva \\
4 & Alcaldía del municipio de Maicao \\
5 & Personería Municipal de Maicao \\
6 & Comsalía General Seccional Riohacha \\
7 & Corporación Autónoma Regional de La Guajira CORPOGUAJIRA \\
8 & Generadora y Comercializadora de Energía Eléctrica del Caribe GECELCA S.A E.S.P. \\
9 & Gases de La Guajira \\
10 & Contraloría Departamental \\
11 & Gobernación del departamento de La Guajira \\
12 & Chevron \\
13 & Sanipublic Asistencia Sanitaria \\
14 & Dirección de Impuestos y Aduanas Nacionales DIAN \\
15 & Interaseo S.A.S \\
\hline Fuente: (Universidad de La Guajira, 2021a)
\end{tabular}

A nivel internacional se destaca la existencia de 43 convenios vigentes (32 convenios marco y 11 específicos), con entidades de países como los Estados Unidos, Republica Dominicana, Perú, España, México, Bolivia, Panamá, Venezuela, Suecia, Argentina, Brasil, Cuba, Ecuador Curazao (Universidad de La Guajira Uniguajira, 2018).

\section{Subcategoría Participación estudiantil en proyectos Universidad-Empresa-Estado}

Se han determinado diversos programas, proyectos y acciones orientados a la vinculación, participación y consolidación de la participación estudiantil en actividades empresariales vinculadas con la universidad y el estado, destacándose las siguientes:

En torno a la Ley 1014 de 2006, de fomento a la cultura del emprendimiento en los estamentos educativos del país, Uniguajira creó la denominada "Unidad de Emprendimiento Centro de desarrollo empresarial (Universidad de La Guajira Uniguajira, 2016). A partir de la cual se ha generado una dinámica de incubación y desarrollo de 
ideas de proyectos empresariales y planes de negocio viables económica, financiera y ambientalmente. A fin de incentivar la vinculación estudiantil con proyectos potenciales desde la universidad para las empresas y el estado.

Actividades de capacitación y sensibilización a los estudiantes, cuyo proceso comienza con la formación de competencias para el emprendimiento y el liderazgo en los estudiantes, orientado por la estructura curricular. Las siguientes fueron las actividades realizadas en este aspecto a los estudiantes y docentes del programa de ingeniería industrial.

\section{Subcategoría Participación estudiantil en programas de innovación}

En materia de la participación de los estudiantes en programas de innovación tales como transferencia de conocimiento, emprendimiento y creatividad, el programa de ingeniería industrial, en concordancia con los esfuerzos de la facultad de ingenierías y a escala institucional, incentiva la vinculación de sus estudiantes y egresados en diversas iniciativas. Especialmente promovidas desde la Oficina de Relaciones Internacionales ORI y socializadas oportunamente por la oficina de comunicaciones de Uniguajira, a través de sus diversos medios digitales, en materia de procesos de intercambio académico y cultural, becas propias y convocatorias externas de carácter internacional (Uniguajira, 2018):

- Intercambio académico y cultural. Uniguajira ha potenciado la participación académica y cultural en países de América, Asia y Europa, a través de la oficina de relaciones internacionales. Mediante el intercambio académico y cultural de 19 estudiantes, docentes y directivos en diferentes países de América, Asia y Europa se convirtió en la universidad destino de cinco pasantes de reconocidas instituciones nacionales y extranjeras.

- Becas propias. Disposición de becas propias a jóvenes para la realización de prácticas profesionales a nivel internacional.

- Programa Delfín. Participación en eventos como la XXIV convocatoria del verano de la investigación científica y tecnológica del pacífico que lidera el Programa Delfín, se logró que 13 estudiantes de Uniguajira realizaran pasantías de investigación en centros de educación superior e institutos científicos mexicanos como la Universidad de Guadalajara, Autónoma de Ciudad Juárez, Autónoma del Estado Morelos, Nacional Autónoma de México, Autónoma de Chiapas, Bemérita Universidad Autónoma de Puebla, el Centro Interdisciplinario de Investigación para el Desarrollo Integral Regional (IPN) de Oaxaca y en los institutos de Ecología (CONACYT) y Ciencias del Mar y Limnología (UNAM).

- Convocatorias externas. Aportes institucionales en materia de financiación externa de actividades de movilidad, capacitación y proyectos de investigación de la comunidad académica. Es por esto, que permanentemente la Oficina de Relaciones Internacionales realiza una extensa búsqueda de becas y convocatorias, las comparten a los estamentos universitarios y asesoran durante su postulación. Universidad de La Guajira entregó tiquetes aéreos y seguros médicos internacionales.

Los resultados del estudio coinciden con los hallazgos de Díaz et al., (2013) y Alvarado (2014) al vincular procesos de mejoramiento continuo, autoevaluación y autorregulación en el nivel de educación superior, como 
factores de fortalecimiento de procesos de desarrollo de los ciudadanos, la sociedad y la humanidad, asociados a la formación para la investigación, el espíritu crítico, la creación. Así como por sus aportes al conocimiento científico, a la innovación y al desarrollo cultural.

\section{CONCLUSIONES}

El programa de Ingeniería Industrial de Uniguajira constituye un programa de pregrado de alta calidad caracterizado por un alto nivel de efectividad en sus procesos de formación para la investigación, el espíritu crítico y la creación. Por sus aportes al conocimiento científico, a la innovación y al desarrollo cultural, se estableció el cumplimiento en alto grado de este programa en este factor 6. Los hallazgos evidencian avances significativos en las actividades asociadas a la característica 29 de este factor.

Respecto a la formación para la investigación, la innovación y la creación artística y cultural (característica 29), el programa se ha destacado por importantes avances en la promoción de la capacidad de indagación y búsqueda, y la formación de un espíritu investigativo, creativo e innovador en sus estudiantes, estableciendo como prioridad formativa la criticidad y el potenciamiento de un pensamiento autónomo para la formulación de problemas de conocimiento y de alternativas de solución, así como la identificación de oportunidades; configurados a través de estrategias articuladas desde el nivel institucional, de la facultad y desde la dirección del programa.

Se destaca la constitución del sistema de investigación institucional, el comité de investigación y extensión de la facultad de ingenierías, los estamentos de la coordinación de investigaciones y extensión de la facultad. Además, del trabajo programático del plan de estudios del programa (revisión, valoración, análisis de la pertinencia y disposición curricular en materia de promoción de la investigación e innovación) a partir de dinámicas internacionales, nacionales e institucionales vigentes. Cabe destacar que este contempla 7 asignaturas específicamente relacionadas con el fortalecimiento y consolidación de una cultura de la investigación, en el sentido de tener entre sus propósitos principales la formación de profesionales competitivos e innovadores, que participen desde las primeras fases y periodos de formación académica en grupos de investigación, semilleros, investigaciones y procesos de indagación científica.

\section{Conflicto de intereses / Competing interests:}

Los autores declaran que no incurre en conflictos de intereses.

\section{Rol de los autores / Authors Roles:}

Francisco Blanchar: conceptualización, curación de datos, análisis formal, adquisición de fondos, investigación, metodología, administración del proyecto, recursos, software, supervisión, validación, visualización, escritura preparación del borrador original, escritura - revisar \& amp; edición.

Yadely Martínez: conceptualización, análisis formal, investigación, metodología, administración del proyecto, recursos, software, supervisión, validación, visualización, escritura - preparación del borrador original, escritura revisar \& amp; edición.

\section{Fuentes de financiamiento / Funding:}

Los autores declaran que no recibió un fondo específico para esta investigación.

\section{Aspectos éticos / legales; Ethics / legals:}

Los autores declaran no haber incurrido en aspectos antiéticos, ni haber omitido aspectos legales en la realización de la investigación. 


\section{REFERENCIAS}

Alvarado, V. (2014). Práctica pedagógica y gestión de aula, aspectos fundamentales en el quehacer docente. Revista UNIMAR, 31(2). http://editorial.umariana.edu.co/revistas/index.php/unimar/article/view/340

Consejo Nacional de Acreditación CNA. (2013). Lineamientos para la Acreditación Institucional. https://www.mineducacion.gov.co/1621/articles-342684_recurso_1.pdf

Consejo Nacional de Acreditación CNA. (2015). Lineamientos para la Acreditación Institucional. http://sig.ucaldas.edu.co/acredita/docs/lineamientosCNA/prog/Lineamientos CNA 2015.pdf

Díaz, L., Torruco, U., Martínez, M., \& Varela, M. (2013). La entrevista, recurso flexible y dinámico. Investigación en Educación Médica, 2(7), 162-167. https://doi.org/10.1016/\$2007-5057(13)72706-6

Ministerio de Ciencias, T. e I. M. (2018). Convocatoria Nacional para el reconocimiento y medición de grupos de investigación, desarrollo tecnológico o de innovación y para el reconocimiento de investigadores del Sistema Nacional de Ciencia, Tecnología e Innovación - SNCTel. https://minciencias.gov.co/convocatorias/investigacion/convocatoria-nacional-para-el-reconocimiento-ymedicion-grupos-0

Sandín, P. (2003). Investigación cualitativa en educación. Fundamentos y tradiciones. Mc Graw and Hill.

Stake, R. (1999). Investigación con estudio de casos (Segunda ed). Morata. https://www.uv.mx/rmipe/files/2017/02/Investigacion-con-estudios-de-caso.pdf

UNESCO. (2009). Conferencia Mundial sobre Educación Superior 2009 - Las nuevas dinámicas de la educación superior y de la investigación para el cambio social y el desarrollo, Paris. https://pep.unc.edu.ar/wpcontent/uploads/sites/46/2017/04/Declaracion_conferencia_Mundial_de_Educacion_Superior_2009.pdf Universidad de La Guajira. (2021a). Convenios académicos y de prácticas empresariales de la Facultad de Ingeniería. https://www.uniguajira.edu.co/financiacion/itemlist/category/71-facultad-de-ingenierias

Universidad de La Guajira. (2021b). Registro de movilidad estudiantil de estudiantes del programa de Ingeniería Industrial. Registro de la oficina de Relaciones Internacionales de la Universidad de La Guajira ORI.

Universidad de La Guajira Uniguajira. (2016). Acuerdo 006 de 2016. Consejo de facultad. Distrito cultural y turístico de Riohacha. https://www.riohacha-laguajira.gov.co/Transparencia/Paginas/normatividad.aspx

Universidad de La Guajira Uniguajira. (2018). Autoevaluación con fines de acreditación del programa de Ingeniería Industrial. https://www.uniagraria.edu.co/wp-content/uploads/2017/06/AutoevaluacionIngenieria-Industrial-2017-.pdf

Vickery, B. (1970). Techniques of Information Retrieval. 